\title{
TEXTUNIVERSALIEN - BETRACHTUNG AN KONKRETEN TEXTEN/TEXTEREIGNISSEN
}

Unter diesem Titel möchte ich zunächst auf die Diskussion über linguistische Universalien vor fast einem halben Jahrhundert zurückgreifen. Damals wurde der Universalienbegriff in die Linguistik, welche sich von der philologischen Sprachwissenschaft absetzte und gern als "modern" apostrophierte, eingeführt. Sie verstand sich als universelle, über einzelsprachliche Besonderheiten hinaus am lingual Wesentlichen interessierte Wissenschaft. Die Hinwendung zur Muttersprache, zu deren innerer Form und zur Deskription als inhaltsbezogener Grammatik wurde als weniger oder gar nicht mehr aktuell empfunden. Struktur und struktural/strukturell erfaßten als Termini statt dessen die leitenden Aspekte des Sprachverständnisses. Am weitesten gehend wurde durch die sog. "TG" die Formulierung der sprachlichen Äußerung als prozessualer Vorgang erklärt. Der ideale Sprecher/Hörer ist danach kompetent, unter Anwendung eines Apparates von Formulierungsregeln sowie entsprechender Auswahl aus einem Inventar von grammatischen Komponenten Sätze zu generieren, auch wenn diese völlig neu und nicht wiederholt sind. Aufgrund des prozessualen Vorgangs ist der Sprecher auch kompetent für die Auflösung einer Ambiguität von oberflächig gleichen Sätzen, indem er diesen differente Tiefenstrukturen zuordnet. Lange blieb die klassische Semantik eine Disziplin der Lexikologie. Die Semantik wurde erst nach einer Phase der Fortentwicklung als ein Aspekt in diese Grammatik eingeführt. Es ist klar, ich habe hier auf Grundzüge des mit dem Namen Noam Chomsky verbundenen Grammatikmodells hingewiesen. Die berühmten Titel markieren die Entwicklung: Syntactic Structures (1957) und Aspects of the Theory of Syntax (1965)/(Dt. 1969). Es ist da von Syntax die Rede, und wirklich zielt diese Grammatik auf die Erklärung der Kompetenz des Sprechers/Hörers für die Formulierung der Sätze, also auf die Erklärung einer Satzkompetenz. Von Text, worauf sich in dieser Studie das Interesse richten soll, ist noch keine Rede.

Die weitere Entfaltung der Grammatik hin zur Erklärung der Textkompetenz trat als Aufgabe der Sprachwissenschaft wegen der Hinwendung zu interdisziplinären Aspekten, die unter den Bezeichnungen Pragmalinguistik, Soziolinguistik und Psycholinguistik laufen, in den Hintergrund. Diese Positionen sind interdisziplinär zu nennen, weil angrenzende Wissenschaften daraufhin befragt werden, was sie zur Lösung von Problemen sprachlicher Form und Funktion oder der Sprachverwendung beitragen können. Es gilt auch die Umkehrung dieser Frage. 
Die Faszination für die "moderne" Linguistik hatte auch ein weitgehendes Zurücktreten klassischer Teildisziplinen der Germanistik wie Namenkunde des Deutschen, Dialektologie des Deutschen oder Sprachgeschichte des Deutschen zur Folge.

In diesem Rahmen der Wissenschaftsgeschichte sollen die Bemühungen um die Erkenntnis sprachlicher Universalien positioniert werden. Das Selbstverständnis der Linguistik als übereinzelsprachlich, als am Phänomen Sprache insgesamt interessiert, begründet natürlich ein universelles Interesse am Sprachlichen. Und wirklich begleitet die Diskussion um sprachliche Universalien und die Suche danach frühzeitig die Einfuihrung oder Rezeption der Linguistik. Folgende Titel und ihr Erscheinungsjahr kennzeichnen diesen Zusammenhang: Joseph H. Greenberg. Ed. (2. Aufl. 1963): Universals of Language. (Bericht eines Kongresses vom April 1961, dessen mitgegebene Teilnehmerliste sehr vielsagend ist); Emmon Bach u. Robert T. Harms. Eds. (1968): Universals in linguistic Theory. (Beiträge zu einem Symposion im April 1967).

Diese Titel können als klassisch bezeichnet werden. Im damit eingeleiteten Diskurs werden die Umrisse einer speziellen linguistischen Disziplin sichtbar, welche darin grundgelegt und repräsentiert ist. In diesem Diskurs über sprachliche Universalien sind die Bemühungen um die Begründung einer Theorie zu erkennen, sowie um die Suche nach einem Inventar sprachlicher Universalien und um die Klassifizierung der erkannten Universalien. In der Übersichtsliteratur vom Typ Lexikon der Sprachwissenschaft oder Linguistisches Wörterbuch wird ein Aufriß über die Ergebnisse konzipiert. ${ }^{1}$ Für die deutsche Sprachforschung sind die Initiativen von Hansjakob Seiler repräsentativ, dokumentiert z.B. in Hansjacob Seiler. Hrsg. (1978), auch dies die Sammlung der Beiträge einer Tagung 1976 in Gummersbach/Köln, und in der beitragsreichen Forschungsübersicht Gunter Brettschneider/Christian Lehmann (1980). In die Sprachtheorie will Eugenio Coseriu (1975) das Universalienproblem einrücken. Zum Standard eines Handbuchs über Universals of Human Language mit vier Bänden endlich hat Joseph H. Greenberg. Ed. (1978) die Ergebnisse dieses Diskurses erhoben. Trotzdem hat die Linguistik diese in ihren Umrissen deutliche Disziplin nicht systematisch eingeordnet, und hat deshalb auch keine Bezeichnung für sie. $^{2}$ Das mag auch damit zusammenhängen, daß man die Thematik bei der herkömmlichen Allgemeinen und Vergleichenden Sprachwissenschaft mit ihren Interessen für die Sprachtypologie, von der auch Greenberg herkommt, angesiedelt sah.

Diese Erörterung über die sprachlichen Universalien muß sich auf einen Begriff beziehen. Unter Universalien sollen die Eigenschaften verstanden werden, welche jede menschliche Sprache besitzt, also Züge oder Merkmale der Sprachstruktur und

1 H. Bußmann (1990); Th. Lewandowski (1990).

2 Man könnte analog zu Linguistische Pragmatik die Bezeichnung Linguistische Universalistik vorschlagen und ihr eine Position im System der linguistischen Einzelaspekte entspr. dem LGL zuweisen. 
Funktionen der Sprache im Leben von Sprachgemeinschaften, die allen Sprachen gemeinsam sind. ${ }^{3}$ Und W. Nöth (1990: 268) faßt sprachliche Universalien mit Bezug auf Universalsprache (Universal Language) fast genauso auf. "Finally, no universal Language project can afford to ignore the results of research in language universals, i.e., those features common to or at least statistically dominant in all languages."

Um dieses anschaulich zu machen, seien einige Universalien aufgezählt, und zwar in dem Bewußtsein, daß diese Qualifizierung hypothetisch bleibt. Diese Nennungen sind so ausgewählt, daß sie für die weitere Argumentation förderlich sind. Die Form der sprachlichen Zeichen ist linear. Diese Feststellung der Linearität der Zeichen ist von de Saussure als Eigenschaft für alle Sprachen angenommen. Die grammatische Organisation aller Sprachen folgt auch dem Prinzip der Zweifachen Gliederung (double articulation), d.h. die bedeutungstragenden Einheiten der Sprachen (Lexeme, Morpheme, Moneme) auf der ersten Gliederungsebene sind auf der zweiten Ebene in eine Folge von Elementarzeichen (Phoneme), welche lediglich bedeutungsdistinktive Funktion besitzen, zerlegbar. Die Grammatik jeder Sprache weist auf den Deskriptionsebenen Inventare der entsprechenden Zeichen aus, die nach Funktionsklassen (Paradigmen) gruppiert sind, z.B. nach Vokalphonemen und Konsonantenphonemen auf der phonemischen Ebene. Die Gegliedertheit der Sprache zwingt zudem, das Wort und die Klassifizierung nach Wortarten aufzufassen und als sprachlich universell zu akzeptieren. Überhaupt kann man die Sprache überall als nach dem Prinzip der Superisation (Superierung) gebildet erklären. Damit ist auf einen Begriff der Allgemeinen Zeichenwissenschaft zurückverwiesen. Man versteht darunter die Fähigkeit, Elemente eines Inventars so zu konfigurieren, daß ein neues, umfassendes Zeichen gebildet wird, das hierarchisch höher steht. ${ }^{4}$ Nach diesem Prinzip kann man hierarchisch aufsteigend die Folge Phonem/Graphem $>$ Wort $>$ Satz $>$ Text vorhersagen, womit als Satz und Text weitere sprachliche Universalien benannt wären. Bezüglich des Satzes liegt durch die Arbeit von Christian Lehmann (1984): Der Relativsatz eine beachtliche Studie der universellen Implikationen vor. Der Nachweis von Textuniversalien muß weiter Anliegen dieser Erörterung bleiben.

Die Superisation kann eigentlich als die zeichenwissenschaftliche Veranschaulichung des Prozesses gelten, welchen W. von Humboldt mit seiner Aussage über die Sprache "Sie muß daher von endlichen Mitteln einen unendlichen Gebrauch machen, und vermag dies durch die Identität der Gedanken und Sprache erzeugenden Kraft" meint. ${ }^{5}$

3 Angelehnt an die Charakterisierung bei Th. Lewandowski (1990).

$4 \quad \mathrm{Zu}$ Superisation (Superierung, Superzeichenbildung) und Superzeichen vgl. Th. Lewandowski (1990).

5 Das Humboldtzitat, hier nach N. Chomsky (1971: 28), stammt aus dessen Werk Über die Verschiedenheit des menschlichen Sprachbaus. Bezeichnend ist die Spannung, die zwischen diesem Titel und der universellen Feststellung im Zitat besteht. 
In der Semiotik gilt die Superisation als konstitutiv für die Sprachzeichenbildung, und geradezu für die Struktur des Textes. "In its internal text syntactic and text semantic structure, the text is characterized as an autonomous and in certain respects closed system. The systemic wholeness of the text is created by a process of integration of individual signs into an integral sign or, in the terminology of information theoretical semiotics, into a supersign. Lotman characterizes this process as follows: 'A text is an integral sign and all the seperate signs of the general linguistic text [i.e., of natural language] are reduced in the text to the level of elements of this sign." 6

Unter anderen aus der Quelle W. v. Humboldt, die er in den Zusammenhang der rationalistischen Sprachwissenschaft stellt, leitet N. Chomsky die Ansicht her, mit seiner Grammatiktheorie universelle Spracheigenschaften aufgefaßt zu haben. Dazu gehört die Auffassung der Sprache als gebunden an eine Kompetenz des Sprechers, und zwar eines idealen Sprechers, womit dann kein konkreter Sprecher gemeint sein kann. Es muß dies idealtypisch der sprachbesitzende und -beherrschende Mensch sein. Ihm eigen ist die psychische Disposition zum Spracherwerb. Chomsky vertritt in diesem Zusammenhang die nativistische Auffassung, jedoch ist diese Disposition auch mit behavioristischer und/oder kognitivistischer Begründung als natürlich, als universell erklärt. Daß die Performanz dann Einzelsprachen zutage bringt, liegt an der kulturellen Differenzierung der Menschheit. $N$. Chomsky dürfte auch für seine Theorie der Satzformulierung universelle Geltung beanspruchen, wie das auch für die Vertreter der Strukturalen Grammatik gelten dürfte, die der Sprache ein Inventar von Grundmustern ihrer Sätze, auf eines von denen sich jeder realisierte Satz beziehen läßt, zusprechen. Damit ist die Bildung des Satzes Möglichkeit, Ziel und Zweck sprachlichen Formulierens. Die linguistische Grammatiktheorie ist bis dahin vornehmlich Syntaxtheorie, die in der Chomskyschen Auffassung kulminierte. Auch bei Greenberg. Ed. (1978) haben wir über die Bände 1. Method and Theory, 2. Phonology, 3. Word Structure eine Aufnahme der Universals of Human Language bis hin zu 4. Syntax. Die Linguistik des Textes war erst in der Entwicklung begriffen, und so hat der Text unter dem Universalienaspekt noch keine Berücksichtigung gefunden.

Empirisch ist aber leicht zu beobachten, daß es den isoliert erscheinenden Satz oder den Satz für sich in der sprachlichen Kommunikation nicht gibt. Der existiert so nur für den Grammatiker. Wenn es um das Kommunizieren von Mitteilungen geht, entstehen sprachliche Äußerungen, welche sich als Zusammenhang von Sätzen konstituieren. Der Satz ist demnach nicht die finale Einheit der sprachlichen Äußerung, sondern dies ist eben der Zusammenhang von Sätzen, welcher sich als Text darstellt. Demgemäß sind Aussagen über die Funktion und den semantischen Beitrag des Satzes vor allem aus Recherchen am Text herzuleiten. Das war implizite schon immer akzeptiert, wie sich durch die Etablierung des Begriffs Kontext erweist. Auch die schon 
erwähnte Superisation als sprachliches Organisationsprinzip fordert heraus, den Satz auf der vorletzten Etage der Hierarchie von Sprachzeichen anzusiedeln und damit die grammatische Realität des Textes auf der höchsten Etage anzuerkennen. So wird der Text zwangsläufig zum Gegenstand der Linguistik. ${ }^{7}$ Zunächst ergibt sich das Ziel, eine Erweiterung der Grammatik vorzunehmen, und zwar mit synthetischer, induktiver Orientierung, bzw. bottom up. Es ist weitgehend erforscht, wie der Satzzusammenhang den Text konstituiert. Die grammatischen Mittel der Anzeige des Zusammenhangs werden unter den Begriffen Kohärenz und Kohäsion etc. erfaßt. Dieser methodische Weg führt zur transphrastischen Konzeption der Textgrammatik.

Die Folgerichtigkeit dieser schrittweisen Expansion der Grammatik läßt sich gut an Problemanalogien veranschaulichen. Es ist früher problematisiert worden, ob ein Wort ein Satz sein könne bzw. umgekehrt. Man hat positiv befunden, und der Terminus Einwort-Satz repräsentiert einen grammatischen Begriff. Analog sollte man nach der Möglichkeit des Einsatz-Textes fragen. Den Nachweis kann man an der Formulierung Lügen haben kurze Beine führen. Sie ist ganz klar ein Satz. Die syntaktische Struktur ist einfach. Wenn man jedoch darüber hinaus schaut, beobachtet man eine weitere Ausgestaltung. Dieser Satz ist aus gleich langen, zweisilbigen Wörtern zusammengesetzt, gestaltet wie ein mit 4 Hebern des Trochäus gemessener Vers. In der Ausgestaltung weist der Satz über seine syntaktische Funktion hinaus. Man kann ihn als lehrhafte Aussage verstehen, als Sentenz, als Lehrsatz also. Es liegt die linguale Konfiguration vor, welche man als Sprichwort auffaßt. Als Sprichwort wird der Form und Funktion nach ein Text bezeichnet, womit die Realität des Einsatz-Textes evident wird. Lügen haben kurze Beine versteht man deshalb nicht als simple Tatsachenfeststellung, die semantisch zudem in sich nicht stimmig wäre, weil die Mitteilung sich durch die Beobachtung des Kotextes erschließt. ${ }^{8}$ Dabei erkennt man einen Zug sprachlichen Handelns in einer bestimmten Situation.

Mit diesem Beispiel wird außer der Weiterförderung der Syntaxlinguistik zur Textlinguistik auch die der Semantik zur Pragmatik verfolgt. Bekannt ist aus dem frühen Rezeptionsgeschehen der Pragmatik in die Linguistik die exemplarische Erklärung der sprachlichen Aufforderungshandlung. In der Äußerung Monika, es zieht respektive Monika, ich wundere mich, daß es dich gar nicht stört, daß das Fenster aufsteht soll unter Auswertung der situativen Verhältnisse, unter denen "kommuniziert" wird, und durch die Berücksichtigung einer erschlossenen Intention des Sprechers

7 Eben weil der Text die finale Einheit der sprachlichen Formulierung ist; vgl. R. Müller (1997: 109f./Anm. 14). Die in diesem Abschnitt vertretenen Auffassungen vom Text treffen sich weitgehend mit den Darlegungen 3. Criteria of Textuality und besonders 3.2.1 TEXT AS MESSAGE WITHIN A CONTEXT bei W. Nöth (1990: 332).

8 Nach R. Müller (1990: 124) ist Kontext der ältere Terminus, der die Zusammenhänge der sprachlichen Formulierung insgesamt umgreift. Mit der Erweiterung des Gegenstandsinteresses in das Gebiet der Linguistik des Textes hat sich die Notwendigkeit einer begrifflichen Erweiterung und Differenzierung ergeben. In einem terminologischen Lexikon werden daher Kontext und Kotext auseinander gehalten; vgl. H. Bußmann (1990, 417f., 427). 
Monika sich aufgefordert fühlen, die Bedeutung "Monika, schließe doch das Fenster" zu verstehen. ${ }^{9}$

Es liegen wieder Äußerungen als Einsatz-Texte vor, aber im komplexeren verbalen Handeln wird man mehr elaborierte Texte formulieren müssen, zumal wenn Expedient und Rezipient nicht gleichzeitig in einer Kommunikationssituation beisammen sind. Ich verweise nur auf das Erzählen eines Witzes oder das Schreiben eines Briefes. Die Etablierung der Textlinguistik erforderte die der linguistischen Pragmatik (et vice verse), so daß man feststellen könnte, die Pragmatik sei Komponente einer Semantik des Textes. ${ }^{10}$

Außer der Verfolgung der Superisation hat es noch einen anderen Weg der Identifizierung des Textes gegeben. So stellt sich bei einer ganzheitlichen Auffassung von Äußerungspassagen heraus, daß sie als ein Text erscheinen oder aber sich dann in Texte gegliedert erweisen, wenn umfassende Konfigurationen vor Augen stehen, etwa die Feuilletonseite einer Zeitung. Diese Perspektive veranlaßt eine analytische, deduktive Auffassung des Textes, bzw. top down, in dem Bewußtsein, daß man ein Superzeichen vor sich hat. ${ }^{11}$

Systematisch wurde diese Perspektive bei der Erforschung der gesprochenen Sprache, also der mündlichen Sprachereignisse, genutzt. ${ }^{12}$ Man konnte als richtig erweisen, daß sich die Unzahl von Einzeltexten typisieren und einer geringeren Anzahl von Textklassen zuordnen lassen. Für das Ergebnis stellte schon die Umgangssprache Bezeichnungen bereit, so: Diskussion, Interview, Reportage, Gespräch, Erzählung etc. Auch im schriftsprachlichen Bereich wurde diese Textklassifizierung betrieben. Für diese Textklassen setzte sich immer mehr der Terminus Textsorte durch. Die Inventarisierung und Systematisierung der Textsorten einer Sprache erweist sich als wesentliche Aufgabe der Textlinguistik, und diese Aufgabe ist nach wie vor aktuell, wie sich an der Thematik des Symposions in Ljubljana 1995 u. 1997 und dem Inhalt vieler Beiträge des Symposions 1995 und des gegenwärtigen (1997) zeigt. ${ }^{13}$

Die kontinuierliche Entwicklung der Textlinguistik läßt sich verfolgen ab dem frühen Aufruf Textlinguistik als linguistische Aufgabe von P. Hartmann in 1968 über den Beitrag Aufgaben und Methoden der Textlinguistik. Kritischer Überblick über den Forschungsstand einer neuen linguistischen Teildisziplin von K. Brinker in 1971 bis zur vollen fachlichen Ausgestaltung in der Gegenwart. ${ }^{14}$ Die Anliegen und Methoden der

Dieses Beispiel stammt aus Funk-Kolleg Sprache, Bd. II (1973: 113-123), 9. Sprechakte von Dieter Wunderlich.

10 Bei R. Müller (1990: 90) wird dieser Zusammenhang von Textlinguistik und Pragmalinguistik hergeleitet.

11 Zum Superzeichen vgl. vorn Zitat bei Anmerkung 6.

12 Forschungsbericht G. Schank u. G. Schoenthal (1983) und Erörterung bei R. Müller (1995: 40ff.)

13 Zu Textsorten vgl. R. Müller (1995); Dokumentation des Symposions 1995 in Linguistica XXXV,1 Textsorten (1995).

14 P. Hartmann (1968) wiederveröffentlicht in W. Dressler Hrsg. (1978). K. Brinker (1971) in Wirkendes Wort 21, 217-237. 
Textlinguistik werden in Handbüchern und Lexika der Linguistik umrissen und mit umfangreichen Bibliographien belegt. Es wird ein differenziertes System von Begriffen mit beispielsweise Text, Textem über Texttheorie, Textsorte, Textualität bis Textwissenschaft ausgebildet. Als Fach in der sprachwissenschaftlichen Lehre weist sie sich durch viele sog. Einführungen in die Textlinguistik aus, z.B. K. Brinker (1988), W. Heinemann u. D. Viehweger (1991), H. Vater (1994), um nur die neueren zu nennen.

Eine gewisse Irritation zeigt sich bei der Einordnung der Textlinguistik in die Linguistik insgesamt, weil sie als Teil der Grammatik, mit dem diese über die Syntax hinaus expandiert, angesehen wird, oder auch als zusätzliche linguistische Disziplin neben Grammatik, Pragmalinguistik, Soziolinguistik, Psycholinguistik, Historiolinguistik etc. Die Perspektive, ob struktural oder funktional, bestimmt wohl die Tendenz der Zuordnung.

Ein Eindruck dieser Unbestimmtheit ergibt sich aus folgendem. Es gibt in der systematischen Übersicht der Linguistik zwei diskrete Bereiche, die bei W. Kürschner (1994: 70f.) folgendermaßen umrissen werden: I. Linguistische Untersuchungs- und Beschreibungsebenen; II. Linguistische Teildisziplinen und angrenzende Wissenschaften. Bei A. Linke, M. Nussbaumer, P.R. Portmann (1994: Inhaltsverzeichnis) erscheinen diese Positionen einfach als Teil I und Teil II. Diese Zweiteilung (Teil I, Teil II) soll hier auch als Grundkonzeption der Linguistik aufgefaßt werden.

Interessant ist, wo die Textlinguistik zugeordnet wird. Bei Funk-Kolleg Sprache, Bd. II (1974) erscheint sie nur implizit, also ohne ausdrückliche Nennung, speziell in den Kapiteln Redekonstellation und Sprachverhalten I, II, obwohl hier ein früher Beleg für den Terminus Textsorte vorliegt. Bei W.A. Koch, Hrsg. (1973/74) wird man sie Teil II zuordnen, während sie im Lexikon der Germanistischen Linguistik (1980) unter "Sprachstrukturen", also eher Teil I, geführt wird. In Kleine Enzyklopädie: Deutsche Sprache (1983) wird der Text sowohl in dem dem Teil I zuzuordnenden Zusammenhang als auch in dem dem Teil II zuzuordnenden behandelt. Bei H.-D. Kreuder (1993) steht die Textlinguistik bei Untersuchungsebenen der Sprache, also Teil I, während Pragmalinguistik bei Aspekte der Sprachbetrachtung, also Teil II, steht. A. Linke u.a. (1994) haben Textlinguistik in Teil II, aber Pragmatik in Teil I. Schließlich stehen bei W. Kürschner (1994) Textlinguistik und Pragmatik zusammen unter Linguistische Untersuchungs- und Beschreibungsebenen, also Teil I. Eine Disziplin Universalienlinguistik oder Universalistik taucht in diesem Horizont der Linguistik gar nicht auf.

Die wissenschaftsgeschichtliche Epoche, in welcher die Sprachwissenschaft hauptsächlich als Philologie der Einzelsprachen betrieben wurde, erfuhr also in den letzten Jahrzehnten oder gar im letzten halben Jahrhundert eine Weiterung zur "modernen" Linguistik. Es ergaben sich neue Aspekte für den Gegenstand Sprache und neue Methoden für die Erforschung der Sprache. Die Linguistik des Textes oder Textlinguistik gehört in den Zusammenhang der Expansion der Grammatik über die Syntax hinaus und wurde auch als ganz neue Disziplin in die Sprachwissenschaft eingebracht. 
Interessant ist, dies im Aufriß der Wissenschaftsgeschichte der Linguistik widergespiegelt $\mathrm{zu}$ finden, wofür folgendes zeugen soll: Nach G. Helbig (1974) kulminiert diese Geschichte, wie auch hier zuvor festgestellt, in der Linguistik von N. Chomsky mit der Generativen Grammatik und der TG, wobei mehr beiläufig auf den Anspruch von Chomsky hingewiesen wird, daß seine linguistische Theorie auch einen Bericht über linguistische Universalien inkorporiere..$^{15}$ Vom Text ist laut Sachregister noch keine Rede, geschweige denn von Textlinguistik. G. Helbig (1990) ist eine Fortsetzung der "Geschichte der neueren Sprachwissenschaft", und zwar mit der Entwicklung seit 1970. Im Zusammenhang mit der sog. kommunikativ-pragmatischen Wende in der Linguistik konstituieren sich die diversen Einzeldisziplinen, von denen die Textlinguistik eine ist. ${ }^{16}$ Von Universalien ist keine Rede mehr, umso mehr aber von Text, sogar Textsorte etc. Gehen wir noch einmal auf die Einordnung der Textlinguistik in den besprochenen Systematiken zurïck, so erweist sich die Textlinguistik bei G. Helbig (1990) in Teil II eingeordnet.

Im Verlauf der linguistischen Orientierung der Sprachwissenschaft wurde auch die Diskussion über sprachliche Universalien geführt, aber ohne daß damit eine Disziplin der Linguistik explizit ausgebildet wurde. Für diese Universaliendiskussion muß eine Ungleichzeitigkeit gegenüber der Entfaltung der Textlinguistik konstatiert werden. Während die Erforschung der sprachlichen Universalien ihre Aktualität einbüßte, konsolidierte sich die Textlinguistik. Gleichzeitig verlief die Universaliendiskussion mit der linguistischen Diskussion der Syntax und der grammatischen Subebenen. Die Beantwortung von Fragen an die Textlinguistik hinsichtlich des Problems der sprachlichen Universalien ist noch nachzuholen.

In diesem Beitrag wird also gefragt, ob der Text und auch die Textsorte als Ergebnis der Klassifizierung des Textaufkommens in der menschlichen Sprache zu den sprachlichen Universalien gehören. In einer empirisch begründeten Darlegung, d.h. am Beispiel von Texten, welche zu den Einfachen Formen gehören, wird hier eine positive Antwort gefunden.

Dem Anliegen entsprechend sind Texte gewählt, deren Herkunft und Fundort sehr unterschiedlich sind, deren Verwendung, Thematik, kommunikative Funktion jedoch als übereinstimmend bzw. einander sehr ähnlich erkannt werden können.

Als erster wird ein sehr bekannter Text, der im alten Testament der Bibel steht, herangezogen. 
Text $1^{17}$

Genesis 111-119

Der Turm von Babel

111 Es hatte aber die ganze Erde die gleiche Sprache und die gleichen Worte. 2 Als sie von Osten aufbrachen, fanden sie eine Ebene im Lande Schinear und ließen sich dort nieder. 3 Sie sprachen zueinander: "Wohlan, wir wollen Ziegel formen und sie brennen!" Der Ziegel diente ihnen als Stein, und das Erdpech diente ihnen als Mörtel. 4 Dann sagten sie: "Wohlan, laßt uns eine Stadt bauen und einen Turm, dessen Spitze bis zum Himmel reicht! Wir wollen uns einen Namen machen, damit wir uns nicht über die ganze Erde zerstreuen!"

5 Da stieg Jahwe herab, um die Stadt und denTurm anzusehen, den die Menschen gebaut hatten. 6 Und Jahwe sprach: "Siehe, sie sind ein Volk und sprechen alle eine Sprache. Das ist erst der Anfang ihres Tuns. Fortan wird für sie nichts mehr unausführbar sein, was immer sie zu tun ersinnen. 7 Wohlan, wir wollen hinabsteigen und dort ihre Sprache verwirren, so daß keiner mehr die Sprache des anderen versteht!" 8 Da zerstreute Jahwe sie von dort über die ganze Erde, und sie mußten aufhören, die Stadt zu bauen. 9 Darum nennt man sie Babel. Denn dort hat Jahwe die Sprache der ganzen Erde verwirrt, und von dort hat sie Jahwe über die ganze Erde zerstreut.

Es ist der Bericht vom Turmbau zu Babel. Er kann nach Mesopotamien in die babylonische Geschichte zurückverfolgt werden, gehört also zu den ganz alten Erzählungen der Menschheit. Für die Tradierung sind viele Sprachen in der Vergangenheit und Gegenwart sowie in unterschiedlichsten Gegenden in Anspruch zu nehmen; zuerst die semitischen mit Akkadisch, Hebräisch, Aramäisch, dann das Griechische und das Lateinische. Eigentlich sind auch die Sprachen beteiligt, in welche die Bibel später übersetzt worden ist. Und das sind so gut wie alle. Hier wird die deutschsprachige Version vorgelegt.

Der zweite Text steht an nicht so repräsentativer Stelle. Er erzählt von der Findung eines besonderen Käses.

\section{Text $2^{18}$}

"Es war einmal vor mehr als 2000 Jahren, als das Schicksal in Gestalt einer geheimnisvollen jungen Frau die Entstehung des Roquefort bewirkte", so sagt die Legende.

Ein Hirte, der gerade sein Mittagsmahl in eine Felsengrotte gelegt hatte, um es ganz frisch zu halten, bemerkte ein junges, wunderschönes Mädchen. Sie zog ihn so sehr an, daß er ihr nacheilte.

Tagelang lief er hinter ihr her, bis sie plötzlich am Horizont der Hochebene verschwand. Als er eines Tages zur Grotte zurückkehrte, fand er dort sein Roggenbrot und den Schafkäse wieder. Aber welcher Schrecken! Das Brot war verschimmelt und der Käse von zartgrünen Äderchen

17 Aus: DIE BIBEL. Die heilige Schrift des alten und neuen Bundes (1965), 20. Auflage, 9. Man hätte auch die Erzählung von der Sintflut o.a. als Text aus der Bibel wählen können.

18 Prospekt der Société des Caves et Producteurs reunis de Roquefort. F-12250 Roquefort-sur-Soulzon, 7. 
durchzogen. Doch da er hungrig war, probierte er von dem Käse. Und ein Wunder war geschehen: Der einfache Schafkäse hatte sich in eine Köstlichkeit verwandelt.

Die Geschichte steht im Prospekt einer französischen Firma, welche diesen Käse produziert und vertreibt. Der Name der Produktionsstätte in Frankreich ist zugleich der Name des Produkts: Roquefort. Man kann annehmen, daß die Erzählung in einer der historischen Sprachen, die man in der Landschaft des Larzac jemals sprach, formuliert wurde. Seit längerer Zeit nun vermittelt man auf Französisch diese Mär. Hier wird eine deutschsprachige Version vorgelegt.

Es wird hier angenommen, daß der Text die Geschichte des Produktes seit langem begleitet und nicht etwa zum Zwecke der Werbung eigens "nachempfunden" wurde. ${ }^{19}$

Der dritte Text stammt aus Hessen. Er präsentiert die Deutung des Namens einer adligen Familie in Nordhessen zum Sächsischen hin: Von der Malsburg.

\section{Text $3^{20}$}

\section{Ursprung der von Malsburg}

Die von der Malsburg gehören zu dem ältesten Adel in Hessen und erzählen: Zur Zeit als Karl der Große den Brunsberg in Westfalen erobert, habe er seine treuen und versuchten Diener belohnen wollen; einen Edelmann, namens Otto, im Feld vor sich gerufen, und ihm erlaubt, daß er sich den Fels und Berg, worauf er in der Ferne hindeute, ausmalen (d.h. eingrenzen, bezeichnen) und für sich und seine Erben eine Festung dahin bauen dürfe. Der Edelmann bestieg den Felsen, um sich den Ort zu besehen, auszumalen und zu beziehen; da fand er auf der Höhe einen Dornstrauch mit drei weißen Blumen, die nahm er zum Mal-, Kenn- und Merkzeichen. Als ihn der König hernach fragte, wie ihm der Berg gefalle, erzählte er, daß er

19 Dieser Gedanke wurde durch einen Text hervorgerufen, der in die aufwendige Werbeanzeige einer Kaffee-Firma mit dem farbigen Kopffoto eines Massai einmontiert ist. Die Analogie zum Roquefort-Text ist frappierend und beweist, daß eine Vergewisserung hinsichtlich dieser Textart und -funktion aus den Einfachen Formen (Legende) besteht, welche diese für veränderte Kommunikationszwecke (Warenwerbung) instrumentierbar macht. Eine Änderung der Pragmatik dieser Textsorte wird so herbeigeführt.

Text 2a:

In Afrika liegt

der Ursprung des Kaffees.

Seine Vorfahren erzählten, ein Pilger habe einst den Kaffe entdeckt.

Er steckte seinen Wanderstock tief in den fruchtbaren Boden Afrikas

um zu beten. Überrascht sah er, wie sich das trockene Holz seines Stockes

in einen blühenden Kaffeestrauch voll roter Früchte verwandelte.

Ein Wunder war geschehen.

Seither reifen in dieser afrikanischen Erde

die Bohnen für einen der besten Kaffees der Welt.

Der würzigste aller Privat Kaffees

läßt dieses Wunder wieder wahr werden: African Blue

20 U. Diederichs u. Ch. Hinze (1978: 77). Der Text ist über die Sagensammlung der Brüder Grimm von 1818 letztlich aus J.J. Winkelmann, Beschreibung der Fürstentümer Hessen und Hersfeld von 1697 überliefert. 
oben einen Dornbusch mit drei weißen Rosen gefunden. Der König aber sonderte ihm sein gülden Schild in zwei gleiche Teile, obenhin einen Löwen und unten drei weiße Rosen. An dem ausgemalten Ort baute Otto hernach seine Burg und nannte sie Malsburg, welcher Name hernach bei dem Geschlecht geblieben ist, das auch den zugeteilten Schild bis auf heute fortführt. (85)

Die Erzählung ist nur von regionalem Interesse. Sie zeugt jedoch von einer allgemeinen Neigung, nämlich den Namen nicht lediglich als identifizierendes Sprachzeichen und bedeutungsarmes Etikett des Trägers aufzufassen, wie die Onomastik es nahelegt, ${ }^{21}$ sondern als Zeugnis, welches eine Erklärung und Würdigung der Namensträger abgibt. Dieses Bedürfnis nach Namensdeutung erscheint übrigens auch als universell. Die Namen werden "beim Wort genommen". Auch der Wappenschild, der den Adel auszeichnet und die Adligen unterscheidet, wird im Rahmen dieser bedeutsamen Sage beschrieben und aufgewertet.

Zuletzt, als vierter Text, sei jener mit der unkonventionellen Interpunktion dokumentiert.

\section{$\operatorname{Text} 4^{22}$}

Erzählung. Thema: Schöpfungssage der Philippino.

es gibt auf den $z+$ Philippinen $+z$ eine $+p+$ alte Geschichte,+ die im Volk erzählt wird,,++ wie die $z+$ Philippinen $+z$ entstanden sind,$++p+$ zunächst gab es nur den Himmel und das Wasser . es gab noch kein Land . aber der Schöpfer hatte auch schon einen Vogel erschaffen . dieser Vogel flog nun zwischen dem Himmel und dem Wasser . nach einigem Flug ermüdete er und suchte ein Plätzchen i+ um sich niederzulassen zu können $+i$. er fand aber kein Land und konnte sich nicht niederlassen . nun sann der Vogel darauf ,+ wie Land geschaffen werden könnte +,,+ wie er einen Platz finden könne,$+ i+$ um sich einmal auszuruhen $+i$. er hetzte zu diesem Zweck das Meer gegen den Himmel auf . das Meer schlug Wogen ,+ die gen Himmel spritzten,+ . / der Himmel wurde darüber ärgerlich und setzte sich nun gegen das Meer zu Wehr ,+ indem vom Himmel herunter Steine geworfen wurden immer und mehr und immer mehr,+ . durch dieses Abwerfen der Steine wurde schließlich das Meer beruhigt . und ,+ nachdem viele Steine abgeworfen waren + , schaute schließlich aus dem Meer an einigen Stellen Land hervor . dieses Land (das erste Land auf dieser Welt) waren die $\mathrm{z}+$ Philippinen $+\mathrm{z}$. es wird dadurch auch erklärt ,+ daß die $z+$ Philippinen $+z$ ein so inselreiches Gebiet sind + , (ein Gebiet ,+ das etwa siebentausend Inseln umfaßt + ,) . denn durch die unregelmäßig abgeworfenen Steine sind nun an verschiedenen Stellen eben einzelne Landstücke aus dem Meer aufgetaucht . und dieses alles zusammen bildet die $z+$ Philippinen $+z$. damit gab es also die $z+$ Philippinen $+z$. aber es gab damit noch nicht den Philippino . wie ist nun dieser Philippino dieser Mensch in die Welt gekommen ? . +p+ die Götter fanden, + daß das Land ,+ was es nun gab +, doch recht einsam leer und unbewohnt sei,+ und sie beschlossen $i+$ den Menschen zu erschaffen $+i$. sie formten

21 Danach haben Namen identifizierende/indizierende Funktion und kaum begriffliche Bedeutung; vgl. R. Müller (1995b: 21).

22 Texte gesprochener deutscher Standardsprache I (1971: 76ff.). Es handelt sich um die Transkription der Tonbandaufnahme aus der Sendung "Zwischen Hamburg u. Haiti" des NDR Hamburg am 22.1.67. 
zu diesem Zweck einen grossen Lehmklumpen . und $+p+$ nun $+p+$ wollten sie diesem Lehmklumpen Leben einhauchen . hierzu schoben sie den Lehm zunächst in einen Backofen i+ um ihn zu backen $+i$. nach einiger Zeit schalteten sie den Ofen ab zogen den Lehmklumpen heraus mußte aber bestürzt feststellen,+ daß sie offenbar zu lange gebacken hatten,+ . der Lehmklumpen war dunkel finster . aber ,+ nachdem sie sich schon einmal die Mühe gemacht hatten + , hauchten sie ihm trotzdem Leben ein.

und so entstand der Schwarze . sie wiederholten den Versuch ,+ da das erste Ergebnis nicht ganz befriedigte + , . sie formten einen neuen Lehmklumpen . sie schoben ihn in den Ofen . sie heizten den Ofen an und waren nun sehr vorsichtig . schon nach kurzer Backzeit zogen sie den Klumpen aus dem Ofen heraus. aber nun mußten sie feststellen ,+ daß sie die Backzeit doch zu kurz gewählt hatten,+ . der Lehmklumpen war sehr hell geblieben . trotzdem auch jetzt (sie hatten sich einmal die Mühe gemacht) hauchten sie auch diesem Klumpen Leben ein . und so kam der Weiße auf diese Welt . bei einem dritten Versuch hatten sie nun genügend Erfahrung gesammelt / der nächste Lehmkloß , + den sie jetzt formten und in $\mathrm{n}$ Ofen schoben + , den buken sie eine $+p+$ genau richtige Zeit . und mit Freuden zogen sie nun einen leicht braunen Klumpen aus dem Ofen heraus . ihm hauchten sie Leben ein . und so entstand der wohlgebackene braunhäutige Philippino.

Es ist eine Geschichte, die ein Reisender im Rundfunk erzählt hat, sie wurde in dieser Art transkribiert, um den mündlichen Stil hervortreten und auch in der schriftlichen Version ansichtig sein zu lassen. Der Reisende bedient sich hier der deutschen Sprache, aber der Inhalt deutet darauf hin, daß es sich um die Erzählung aus einer fernöstlichen Kultur handelt, welche zuerst in einer dort gebräuchlichen Sprache formuliert worden sein wird, vielleicht in der indonesischen Sprache Tagalog, von dem die Amtssprache der Philippinen unter dem Namen Pilipino hergeleitet ist. So auf Deutsch kommt sie sprachlich aus zweiter, vielleicht auch dritter Hand, wenn diese paradoxe Metapher hier einmal gebraucht werden darf.

Bei diesen vier Texten soll es zunächst belassen werden. Es wurde die Diversifikation ihres Herkommens in mehrfacher Hinsicht, der Sprache, der Region, der Zeit, vorgenommen.

Die Diversifikation ist aber gegen den Eindruck durchzusetzen, daß sich die vier Beispieltexte in wesentlichen Zügen auch gleichen. Da ist die Mitteilung des episodischen Inhalts und die sprachliche Reproduktion eines fiktionalen Geschehnisses, welche in konzentrierter Prosaformulierung mitgeteilt wird. Als Form haben sich narrative Texte angeboten, für die die deutsche Umgangssprache schon die bisher gebrauchten Benennungen eine Geschichte, eine Erzählung bereitstellt.

Hier zeigt sich, daß Texte die finalen Gebilde der sprachlichen Äußerung sind. Früher ist das schon am Sprichwort, welches musterhaft als Ein-Satz-Text gebildet ist, angesprochen worden. Es soll mit der Wertung als finales Gebilde darauf hingewiesen werden, daß realistische Äußerung in Sprache überhaupt nur als Text erfolgt, und um auch damit zu zeigen, daß der Text an sich sprachliche Universalie ist. Das war auch schon vorherzusagen, als die Erkenntnis des superierten Aufbaus der Sprache eine transphrastische Ebene der grammatischen Deskription, die Textebene, verhieß. Als deren funktionale und formale Einheit muß der Text begriffen werden. Sein theoretisches Gewicht als sprachliche Universalie rechtfertigt die Textlinguistik als 
sprachwissenschaftliche Disziplin, welche die Existenzbedingungen des Textes erforscht und darstellt.

Für eine weitere Auskunft werden die Texte wieder herangezogen, und zwar geht es um das für alle vier Gemeinsame und Übereinstimmende. Alle vier beschäftigen sich mit auffälligen Gegebenheiten, deren reale Existenz oder deren Herkunft bzw. Entstehung rational nicht erwiesen werden kann. Im ersten Text ist diese Gegebenheit die leidige Teilung der Menschheit in Völker mit unterschiedlichen Sprachen; im zweiten die erstaunliche Bindung des Roquefort und das Heranreifen des Käses durch Zusammenwirken von Mensch und Natur an einem bestimmten Herstellungsort, der landschaftlich exponiert ist. Im dritten Text ist es die Gegebenheit des hervorragenden Standes, der begüterten Ausstattung und der repräsentativen Wohnverhältnisse einer Familie mit Tradition, was alles begründet und legitimiert zu werden beansprucht. Der vierte Text zeugt von der Faszination der Philippinos von dem Archipel, der ihre Heimat ist, und von der Überzeugung, daß sie eigens als Bewohner dieses inselreichen Gebietes geschaffen wurden. Als weitere Auffälligkeit beachten die Philippinos ihre ideale Hautfarbe, die sich von den Extremen Schwarz und Weiß wohltuend unterscheide. Es handelt sich wohl um die Kontamination zweier Geschichten, von denen die zweite jünger ist und aus der Begegnung mit Menschen weißer und schwarzer Hautfarbe resultiert. Die Gegebenheiten, die für die Philippinos geheimnisvoll bzw. rätselhaft sind, liegen auf der Hand.

Nimmt man die Zuordnung der vier Geschichten zu einer Textsorte vor, so wird zum Argument, daß die narrative Kurzform und die Funktion, eine Erklärung für nicht beweisbare Gegebenheit beizubringen, konvergieren. Das Ereignis, das die Erklärung bietet, wird in mythische Zeiten verlegt. Die Funktion, solcher Art Erklärungen zu schaffen, ist exemplarisch einer Textsorte zugeschrieben, der Sage. Dem würde auch A. Ohler (1986) beipflichten, die die auch hier herangezogene Turmbauerzählung aus dem Pentateuch in den methodischen Schritten der Literarkritik, der Textkritik und der Gattungskritik der Sage zuordnet. Die Turmbauerzählung erfülle weithin die Bedingung: "In Sagen sprechen Menschen, die von einer Erfahrung so beeindruckt sind, daß sie sie weitergeben wollen, die Hörer sind wiederum von der Erzählung so angetan, daß sie sie weitersagen, so entsteht die Sage, und so lebt sie fort: im Weitersagen", so daß sie die Turmbauerzählung weder historischen Bericht noch Mythos nennen will, sondern am ehesten eine Sage. ${ }^{23}$ Gleiches kann man für die drei anderen Beispieltexte in Anspruch nehmen. ${ }^{24}$

Das Klassifizierungsergebnis ist also, daß die vier Texte Sagen sind. Aus dieser Feststellung muß man zugleich auf die Existenz einer Textsorte schließen. Für die Sage ist dies bekannt. So wird sie von A. Jolles (1958) unter den Einfachen Formen

23 A. Ohler (1986: 29-34; Zitat 32).

24 Bei den Beispieltexten 2 und 2a (Anm. 19) tendiert man wegen des Frömmigkeitstopos "Wunder" zur Einschätzung als Legende, eine der Sage als Einfache Form nächstverwandte Textsorte. Es dominieren m.E. jedoch die Kriterien für Sage. 
aufgeführt und gedeutet. Eine Subklassifizierung wird von L. Röhrich (1971) vorgeschlagen. Eine Subklasse "Erklärungssagen", denen die vier Textbeispiele zu subsumieren wären, ist dort isoliert. ${ }^{25} \mathrm{Da}$ die Identifizierung dieser Textsorte das Ergebnis von Textklassifikation ist, muß man damit rechnen, daß sie eine unter mehreren ist. Ein Textsorteninventar ist also zu konstatieren. Was hier für die eine Textsorte Sage demonstriert ist, hat man auch für Textsorteninventare anzunehmen, nämlich die Existenz in vielen weit voneinander entfernt existierenden Sprachen. Textsorte muß man also als sprachliche Universalie erkennen, und zwar in dem Sinne, $\mathrm{da} B$ jeder realisierte Text in irgendeiner Sprache zu einer Textsorte gehört. Der Terminus Textsorte steht für einen Begriff, hier z.B. für den alle Sagen umfangenden. Man kann die Textsorte als Universalie auffassen, ohne daß die Textsorteninventare verschiedener Sprachen übereinstimmen müßten. Die Textsorteninventare sind kulturbedingt und kulturabhängig, sowohl was den Umfang des Inventars, als auch was dessen Differenzierung betrifft. Mit der Veränderung und Entwicklung von Sprachkultur gehen Textsorten verloren, und neue entstehen.

Das Ergebnis dieser Studie im Rahmen des übergreifenden Themas "Textsorten in der interkulturellen Kommunikation" konnte erreicht werden, indem Nachweise an Texten geführt wurden, die eine weite interkulturelle Verbreitung versprechen. Diese sollten zu Texten einer Textsorte, die schon in einfachen und ursprünglichen Kulturen vorhanden und damit weit verbreitet sind, also zu einer archaischen, am besten aus oraler Erzähltraditon stammenden gehören. Dies alles ist für Sagen der Fall. Sie gehören zudem zu den Texten, welche in großer Zahl existieren und, wie bei den Einfachen Formen zugehörigen typisch, in Sammlungen publiziert werden. Die Textsorte "Sage" wird mit H. Bausinger (1980) den Erzählformen der "Volkspoesie" subsumiert, ${ }^{26}$ was auch einen übereinzelsprachlichen Aspekt bietet und für die interkulturelle Verbreitung der Textsorte spricht.

Die Allgegenwart der Textsorte "Sage" in den Sprachen erlaubt, sie zu den sprachlichen Universalien zu zählen. Somit lassen sich alle anderen Textsorten und der Begriff Textsorte, als sprachliche Universalien, als Textuniversalien auffassen.

\section{Literatur}

Bach, Emmon u. Harms, Robert T., Eds. (1968): Universals in Linguistic Theory. London, New York, Sidney, Toronto.

Bausinger, Hermann (1980): Formen der "Volkspoesie". 2. Auflage. Berlin.

26 H. Bausinger (1980: 179-195). Zur Standortbestimmung der Texte oraler Tradition in der Dichtung vgl. auch L. Röhrich u. E. Lindig. Hrsg. (1998). 
Brettschneider, Gunter u. Lehmann, Christian, Hrsg. (1980): Wege zur Universalienforschung. Sprachwissenschaftliche Beiträge zum 60. Geburtstag von Hansjakob Seiler. Tübingen.

Brinker, Klaus (1988): Linguistische Textanalyse. Eine Einführung in Grundbegriffe und Methoden. 2. Auflage. Berlin.

Bußmann, Hadumod (1990): Lexikon der Sprachwissenschaft. 2. Auflage. Stuttgart.

Chomsky, Noam (1957): Syntactic Structures. The Hague.

Chomsky, Noam (1969): Aspekte der Syntax-Theorie. Frankfurt/M., Berlin.

Chomsky, Noam (1971; engl. Original 1966): Cartesianische Linguistik. Tübingen.

Coseriu, Eugenio (1975): Die sprachlichen (und die anderen) Universalien. In: Schlieben-Lange, Brigitte, Hrsg.: Sprachtheorie. Hamburg.

DIE BIBEL. Die heilige Schrift des alten und neuen Bundes (1965), 20. Auflage. Freiburg i. Br.

Diederichs, Ulf u. Hinze, Christa (1978): Hessische Sagen. Düsseldorf, Köln.

Dressler, Wolfgang, Hrsg. (1978): Textlinguistik. Darmstadt.

Funk-Kolleg Sprache. Eine Einführung in die moderne Linguistik. Band II (1973). Frankfurt/M.

Greenberg, Joseph H., Ed. (1963): Universals of Language. 2. Auflage. Cambridge/ Massachusetts und London/England.

Greenberg, Joseph H. (1976): Language Universals. The Hague u. Paris.

Greenberg, Joseph H., Ed. (1978): Universals of Human Language. 4 Bde. Stanford.

Heinemann, Wolfgang u. Viehweger, Dieter (1991): Textlinguistik. Eine Einführung. Tübingen.

Helbig, Gerhard (1974): Geschichte der neueren Sprachwissenschaft. Reinbek bei Hamburg.

Helbig, Gerhard (1990): Entwicklung der Sprachwissenschaft seit 1970. 2. unveränderte Auflage (1988, Leipzig). Opladen.

Jolles, André (1958): Einfache Formen. 2. Auflage (1. Auflage 1930). Darmstadt.

Kleine Enzyklopädie: Deutsche Sprache. Hrsg. von Schildt, Joachim (Federführung) u.a. (1983). Leipzig.

Koch, Walter A., Hrsg. (1973/74): Perspektiven der Linguistik I und II. Stuttgart.

Kreuder, Hans-Dieter (1993): Studienbibliographie Linguistik. 3. Auflage. Stuttgart.

Kürschner, Wilfried (1994): Taschenbuch Linguistik. Berlin.

Lehmann, Christian (1984): Der Relativsatz. Tübingen.

Lewandowski, Theodor (1990): Linguistisches Wörterbuch. 3 Bde. 5. Auflage. Wiesbaden.

Lexikon der Germanistischen Linguistik/LGL. Hrsg. von Althaus, Hans Peter; Henne, Helmut; Wiegand, Herbert Ernst (1980): Studienausgabe I-IV. 2. Auflage. Tübingen.

Linguistica XXXV,1 - Textsorten (1995): Sammelband zu den Beiträgen des internationalen Symposions PROTOTYPISCHES IN TEXTSORTEN Ljubljana 1994. Ljubljana.

Linke, Angelika u. Nussbaumer, Markus u. Portmann, Paul R. (1994): Studienbuch Linguistik. 2. Auflage. Tübingen. 
Methodologische Aspekte der Semantikforschung: Beiträge der Konferenz "Methodologische Aspekte der Semantikforschung" an der Universität Koblenz-Landau/ Abteilung Landau (1996). Hrsg. von Inge Pohl (1997). Frankfurt/M., Berlin, Bern etc.

Müller, Rolf (1990): Einführung in die Semantik. Bedeutung und Bezeichnung, Verstehen und Meinen. Kassel (demnächst bei Langenscheidt in Berlin, München etc.).

Müller, Rolf (1995): Textsorten - Natürliche Sprachformen oder kulturelle Sprachformen? In: Linguistica XXXV,1. Ljubljana, 37-51.

Müller, Rolf (1995b): Möglichkeiten der Kategorisierung von Bedeutung. In: Pohl, Inge, Hrsg.: Semantik von Wort, Satz und Text, 15-24.

Müller, Rolf (1997): Die Erfassung des sprachlichen Zeichens - Von F. de Saussure zu K. Bühler. In: Methodologische Aspekte der Semantikforschung: Beiträge der Konferenz "Methodologische Aspekte der Semantikforschung" an der Universität Koblenz-Landau/Abteilung Landau (1996), 101-114.

Nöth, Winfried (1990): Handbook of Semiotics. Bloomington and Indianapolis.

Ohler, Annemarie (1986): Grundwissen Altes Testament: ein Werkbuch. Bd. 1. Pentateuch. Stuttgart.

Pohl, Inge, Hrsg. (1995): Semantik von Wort, Satz und Text. Beiträge des Kolloquiums in Rostock 1994. Frankfurt/M., Berlin, Bern u.a.

Röhrich, Lutz (1971): Sage. 2. Auflage. Stuttgart.

Röhrich, Lutz u. Lindig, Erika, Hrsg. (1989): Volksdichtung zwischen Mündlichkeit und Schriftlichkeit. Tübingen.

Schank, Gerd und Schoenthal, Gisela (1983): Gesprochene Sprache. Eine Einführung in Forschungsansätze und Analysemethoden. 2. Auflage. Tübingen.

Schlieben-Lange, Brigitte, Hrsg. (1975): Sprachtheorie. Hamburg.

Seiler, Hansjakob, Ed. (1978): Language Universals. Tübingen.

Texte gesprochener deutscher Standardsprache I (1971). Erarbeitet im Institut für deutsche Sprache, Forschungsstelle Freiburg i. Br., München, Düsseldorf.

Vater, Heinz (1994): Einführung in die Textlinguistik. 2. Auflage. München.

Zusammenfassung

Die Suche nach Textuniversalien ist ein Desiderat des linguistischen Diskurses. Mit ihrem Interesse für übereinzelsprachliche Eigenschaften verschafften sich die Linguisten grammatische Erkenntnisse, welche in der Syntaxtheorie und der Erschließung des LAD (language acquisition device) bei N. Chomsky kulminierten. Zugleich verlief separat die Suche nach sprachlichen Universalien, welche sich als erfolgreich erwies und zur handbuchmäßigen Darstellung der Ergebnisse führte. Als Universalien werden die Eigenschaften und Funktionen verstanden, welche allen Sprachen oder zumindest den allermeisten Sprachen eigen sind. Die Universalienforschung verebbte in der Phase der linguistischen Wissenschaftsgeschichte, als die Grammatikforschung mit ihrem Interesse über den Satz hinaus zum Text hin expandierte. Die Wissenschaft vom Text, Textlinguistik und Textpragmatik, wurde etabliert. Thre Haupterkenntnisse liegen in der Erfassung des Gegenstandes Text als finale Einheit der sprachlichen Äußerung, in der Identifizienung der transphrastischen Zusammenhänge der Textstruktur, was zur Textgrammatik führt, und in der Entwicklung des Begriffes Textsorte im "emischen" Auffassungsbereich für das Sprachsystem. Im wissenschaftsgeschichtlichen Kontext wird die Ungleichzeitigkeit der Universalien- und der Textforschung festgestellt, so daß die Frage nach Textuniversalien noch aussteht und Angelegenheit dieser Studie ist. Die Fragestellung wird anhand von vier Sagen, also vier zu den Einfachen Formen gehörenden und 
allenthalben vorfindlichen Texten mit bestimmter Funktion, abgehandelt. Im Ergebnis erweist sich der Text als sprachliche Universalie, und zwar in seiner Funktion als finale Einheit der sprachlichen Formulierung, und als Universalie erweist sich auch der Begriff der Textsorte, unter dem Texte gleicher oder ähnlicher Funktion zusammenzufassen sind, wie hier die Sagen.

\section{Povzetek \\ BESEDILNE UNIVERZALIJE - OB KONKRETNIH BESEDILIH/BESEDILNIH POJAVIH}

Iskanje besedilnih univerzałij je deziderat jezikoslovnega diskurza. S zanimanjem za lastnosti, ki niso značilne le za en jezik, so si jezikoslovci priskrbeli slovnična spoznanja, ki so v skladenjski teoriji in določevanju LAD (language acquisition device) dosegla vrhunec pri N. Chomskyju. Hkrati je ločeno potekalo iskanje jezikovnih univerzalij, ki se je izkazalo kot uspešno in pripeljalo tudi do prikazov v priročnikih. Kot univerzalije razumemo lastnosti in funkcije, ki so lastne vsem jezikom ali vsaj večini jezikov. Raziskovanje univerzalij je uplahnilo v fazi lingvistične znanstvene zgodovine, ko se je raziskovanje slovnice razširilo od stavka na besedilo. Uveljavila se je znanost o besedilu, besedilna lingvistika in besedilna pragmatika. Njena glavna spoznanja temeljijo $v$ pojmovanju predmeta besedilo kot dokončna enota jezikovnega izreka, $v$ identifikaciji transfrastičnih povezav besedilne strukture, kar vodi $\mathrm{v}$ besedilno slovnico, in $\mathrm{v}$ razvoju pojma besedilna vrsta $\mathrm{v}$ distinktivnem področju pojmovanja za jezikovni sistem. V znanstvenozgodovinskem kontekstu je ugotovljena neistočasnost raziskovanja univerzalij in besedila, tako da vprašanje besedilnih univerzalij še ni rešeno in je predmet pričujoče študije. Vprašanje je obravnavano s primeri štirih sag, torej štirih besedil z določeno funkcijjo, ki spadajo med enostavne oblike in jih je mogoče najti vsepovsod. Rezultat študije je besedilo kot jezikovna univerzalija, in sicer $v$ svoji funkciji kot dokončna enota jezikovnega oblikovanja, rezultat pa je tudi pojem besedilne vrste, ki zajema besedila $z$ enako ali podobno funkcijo, kot so tukaj sage. 